\title{
High-resolution imaging of Galactic massive stars with AstraLux
}

\section{138 fields with $\delta>-25^{\circ \star}$}

\author{
J. Maíz Apellániz ${ }^{1,2, \star \star}$ \\ 1 Instituto de Astrofísica de Andalucía-CSIC, Glorieta de la Astronomía s/n, 18008 Granada, Spain \\ e-mail: jmaiz@iaa.es \\ 2 Ramón y Cajal Fellow
}

Received 11 March 2010 / Accepted 20 April 2010

\section{ABSTRACT}

\begin{abstract}
Context. Massive stars have high-multiplicity fractions, and many of them have still undetected components, thus hampering the study of their properties.

Aims. I study a sample of massive stars with high angular resolution to better characterize their multiplicity.

Methods. I observed 138 fields that include at least one massive star with AstraLux, a lucky imaging camera at the $2.2 \mathrm{~m}$ Calar Alto telescope. I also used observations of 3 of those fields with ACS/HRC on HST to obtain complementary information and to calibrate the AstraLux data. The results were compared with existing information from the Washington Double Star Catalog, Tycho-2, 2MASS, and other literature results.

Results. I discover 16 new optical companions of massive stars, the majority of which are likely to be physically bound to their primaries. I also improve the accuracy for the separation and magnitude difference of many previously known systems. In a few cases the orbital motion is detected when comparing the new data with existing ones and constraints on the orbits are provided.

Conclusions. The analysis indicate that the majority of the AstraLux detections are bound pairs. For a range of separations of $0{ }^{\prime} 1-14^{\prime \prime}$ and magnitude differences lower than 8 , I find that the multiplicity fraction for massive stars is close to $50 \%$. When objects outside those ranges are included, the multiplicity fraction should be considerably higher.
\end{abstract}

Key words. techniques: high angular resolution - surveys - astrometry - binaries: visual - stars: early-type - stars: massive

\section{Introduction}

Massive stars play a crucial role in the dynamical and chemical evolution of galaxies. They are the major source of ionizing and UV radiation and, through their huge mass-loss rates, they have a strong mechanical impact on their surroundings. Massive stars are also important because they are critical contributors to stellar and explosive nucleosynthesis. The nuclear products are ejected into space in the stellar winds and in the final supernova explosions that put an end to the massive stars' lives. Despite their importance, our understanding of these objects and of their evolution is still fragmentary due to their relatively small numbers, the existence of unresolved multiple systems, and to their concentration along the Galactic plane, where extinction affects their detection and the measurement of their distances and other properties.

A major ongoing project, the Galactic O-Star Spectroscopic Survey, GOSSS (PI: J. Maíz Apellániz, see Walborn et al. 2010; Gamen et al. 2008, Sota et al. in prep.), is currently obtaining ground-based spectroscopy of all known Galactic O stars with

* Table 3 is also available in electronic form at the CDS via anonymous ftp to cdsarc.u-strasbg.fr $(130.79 .128 .5)$ or via http://cdsweb.u-strasbg.fr/cgi-bin/qcat?]/A+A/518/A1

$\star \star$ Based on data obtained with the $2.2 \mathrm{~m}$ telescope at Calar Alto Observatory (CAHA). Also, some images from the NASA/ESA Hubble Space Telescope (HST) were used. The HST data were obtained at the Space Telescope Science Institute, which is operated by the Association of Universities for Research in Astronomy, Inc., under NASA contract NAS 5-26555.
$B<13$ with the purpose of remedying some of the gaps in our knowledge of Galactic massive stars. All stars are being observed at $R \sim 3000$ to obtain accurate spectral classifications, and subsamples are being observed at $R \sim 40000$ at multiple epochs to detect spectroscopic binaries and at $R \sim 1500$ to accurately measure their spectral energy distributions. The detection of spectroscopic binaries is especially important for massive stars because the multiplicity fraction among them is especially high (Mason et al. 1998), a fact that the preliminary GOSSS results are confirming and even increasing in value. Undetected binaries complicate the study of samples of stars because they introduce biases in the results and lead to incorrect conclusions. The presence of spatially unresolved components can alter the observed spectral type and, in some circumstances, yield lineratio combinations that cannot be present in single $\mathrm{O}$ stars (e.g. different spectral types may be deduced from the He and the $\mathrm{N}$ lines, see e.g. Walborn et al. 2002). Therefore, correctly characterizing the multiplicity can shed light on objects with such composite spectra.

Time-resolved spectroscopy is crucial to detecting shortperiod multiple systems. However, many massive binaries are known to have long periods (Mason et al. 1998) and it has been proposed that their period distribution may follow Öpik's law (Öpik 1924), which would lead to essentially all massive stars being born in multiple systems. To verify that assertion for large-separation systems, spectroscopy is of little use because the periods involved are thousands of years or more and the velocity changes quite small. Therefore, one needs to use high angular-resolution methods in order to visually detect 
companions. Even so, a gap still exists between the two ranges easily detected by spectroscopic and high angular-resolution methods. For example, a $30 M_{\odot}+20 M_{\odot}$ in a circular edgeon orbit with $a=50 \mathrm{AU}$ at a distance of $1 \mathrm{kpc}$ would have a maximum separation of 50 mas and a period of 50 years, both near or beyond the limit of current capabilities. Making the orbit characteristics, mass ratio, or orientation more unfavorable or placing the system at longer distances will make it even harder to detect, to the point that most LMC massive binaries are likely to be currently unknown.

The likely distribution of binary periods (or separations) among massive stars led me to start a project to observe as many massive stars as possible using high-resolution imaging in order to complement the multiple-epoch spectroscopy obtained with GOSSS. Such a systematic project is needed to eliminate biases in our knowledge of massive stars. Different imaging techniques have been attempted to observe binary systems: speckle interferometry, imaging from space, and adaptive optics, among them. Some of the recent attempts are finding previously undetected pairs with large magnitude differences, $\Delta m$ (Turner et al. 2008; Maíz Apellániz et al. 2010), thus opening a new part of the parameter space. In this paper I explore the use of lucky imaging, a technique that, to my knowledge, has not been systematically applied to massive stars. In future papers I plan to extend the sample to include several hundred more stars.

\section{Data}

\subsection{AstraLux}

The main part of the data for this paper was obtained with AstraLux (Hormuth et al. 2008), a lucky imaging camera at the Calar Alto 2.2-m telescope. AstraLux uses an electronmultiplying high-speed CCD capable of obtaining large numbers (typically, 10 000) of short-exposure (typically, $30 \mathrm{~ms}$ ) images of a field of view of $24^{\prime \prime} \times 24^{\prime \prime}$. The images are then combined by a pipeline that selects the $1-10 \%$ of the frames with best seeing and combines them with a drizzle-type algorithm (Fruchter \& Hook 2002). Under good seeing conditions, AstraLux can reach the diffraction limit in the SDSS $i$ and $z$ bands and significantly improve the angular resolution in the rest of the visible part of the spectrum.

The original goal of this project was to observe with AstraLux the $185 \mathrm{O}$ stars with $\delta>-25^{\circ}$ in the Galactic O-Star Catalog of Maíz Apellániz et al. (2004). To that purpose, I observed for ten nights between 12 November 2007 and 10 May 2009 , in all cases using $30 \mathrm{~ms}$ exposures in the SDSS $z$ band and adjusting the detector amplifier to maximize the $\mathrm{S} / \mathrm{N}$ without reaching saturation. Unfortunately, I could observe for less than half of the allocated time because of bad weather and poor seeing, leaving only 138 observed fields. Those include a few massive stars not in the original catalog but present in its second version (Sota et al. 2008), as well as some WR stars. In most cases, a single massive star was known to exist in the field and the AstraLux field was centered on it. For some fields, the primary object was positioned off-center in order to include previously known companions. The effective search radius for companions as measured from the brightest star in a given field is $13^{\prime \prime} 6$, but given the uneven coverage in position angle some companions were found out to $26^{\prime \prime}$. The effective central wavelength for the observations is $9115 \AA$ (with variations of $\pm 5 \AA$ as a function of the airmass) and the bandpass is $1250 \AA$ (defined as the wavelength range containing $68 \%$ of the total throughput).
Each field was processed at Calar Alto using the existing instrument pipeline. In order to maximize angular resolution, I selected for the output images just the $1 \%$ of the frames with better seeing. Observations of the Orion Trapezium $\left(\theta^{1}\right.$ Ori) were used to measure the geometric distortion of the images, with the reference positions of the Trapezium stars measured from an archival WFPC2/HST image. Given the relatively small number of stars visible in the AstraLux Trapezium images, only the linear terms of the geometric distortion were obtained. The fit residuals for the Trapezium stars and AstraLux observations of a comparison field, Cyg OB2-22, for which I had ACS/HRC images (see below), were used to estimate the uncertainties in separation and position angle of the observations. The geometric distortion of the pipeline output images was then removed to produce the final astrometrically corrected images (Fig. 1).

The AstraLux image of a single star observed under good $(\sim 0.7)$ or intermediate $\left(\sim 1^{\prime \prime} .0\right)$ seeing conditions consists of a core that is near-diffraction limited and a halo that is determined by the seeing conditions. The PSF does not appear to show significant variations over the field of view. Since the goal here is to accurately determine the separations and magnitude differences ( $\Delta m$, in this case, $\Delta z$ ) for the pairs observed in each field, I wrote a PSF-fitting program in IDL to simultaneously calculate the positions and fluxes of all detected stars. The PSF itself was fitted using a model that consists of a Gaussian core and a Moffat halo (Moffat 1969). For both components (core and halo), I allowed for the profile to have an elliptical shape in the image plane. When determining the fluxes for each component, I considered both the random uncertainties produced by the PSF-fitting program and the systematic uncertainties resulting from the differences between the real PSF and the fitted profile. The reported uncertainties in $\Delta m$ include both effects. Also, for those cases consisting of a close small- $\Delta m$ binary and a third bright component I did aperture photometry checks to evaluate possible systematic effects in the PSF fitting.

Of the 138 fields observed, I detected a single star in the AstraLux images in 72 fields and two or more stars in the other 66. Given the PSF complexity, I found that visual inspection was the most effective detection mechanism. The systems with a single detected star are given in Table 1. For the systems where two or more stars are detected in the AstraLux data, the measurements for the detected pairs are shown in Table 3. In all cases I searched the Washington Double Star Catalog (WDS, Mason et al. 2001, the used version was downloaded on 10 December 2009) and the Tycho-2 (Høg et al. 2000) and 2MASS (Skrutskie et al. 2006) databases for possible known counterparts of the detected sources.

Table 3 lists a total of 142 pairs, of which 33 were not listed in the WDS. For the new components I have followed a nomenclature consistent with that in the WDS. Seventeen of the 33 pairs not in the WDS had both components in the 2MASS database, leaving 16 components that will be considered strictly new in this paper.

\section{2. $A C S / H R C$}

Three of the fields, Cyg OB2-22, Cyg OB2-7, and 15 Mon, observed with AstraLux were also observed with ACS/HRC on $\mathrm{HST}^{1}$. The Cyg OB2 observations are part of GO program 10602, of which the author is the P.I., and that had as its goal

\footnotetext{
1 Note that there are relatively few HST images of Galactic bright stars with an exposure time short enough not to saturate the central part of the object PSF.
} 


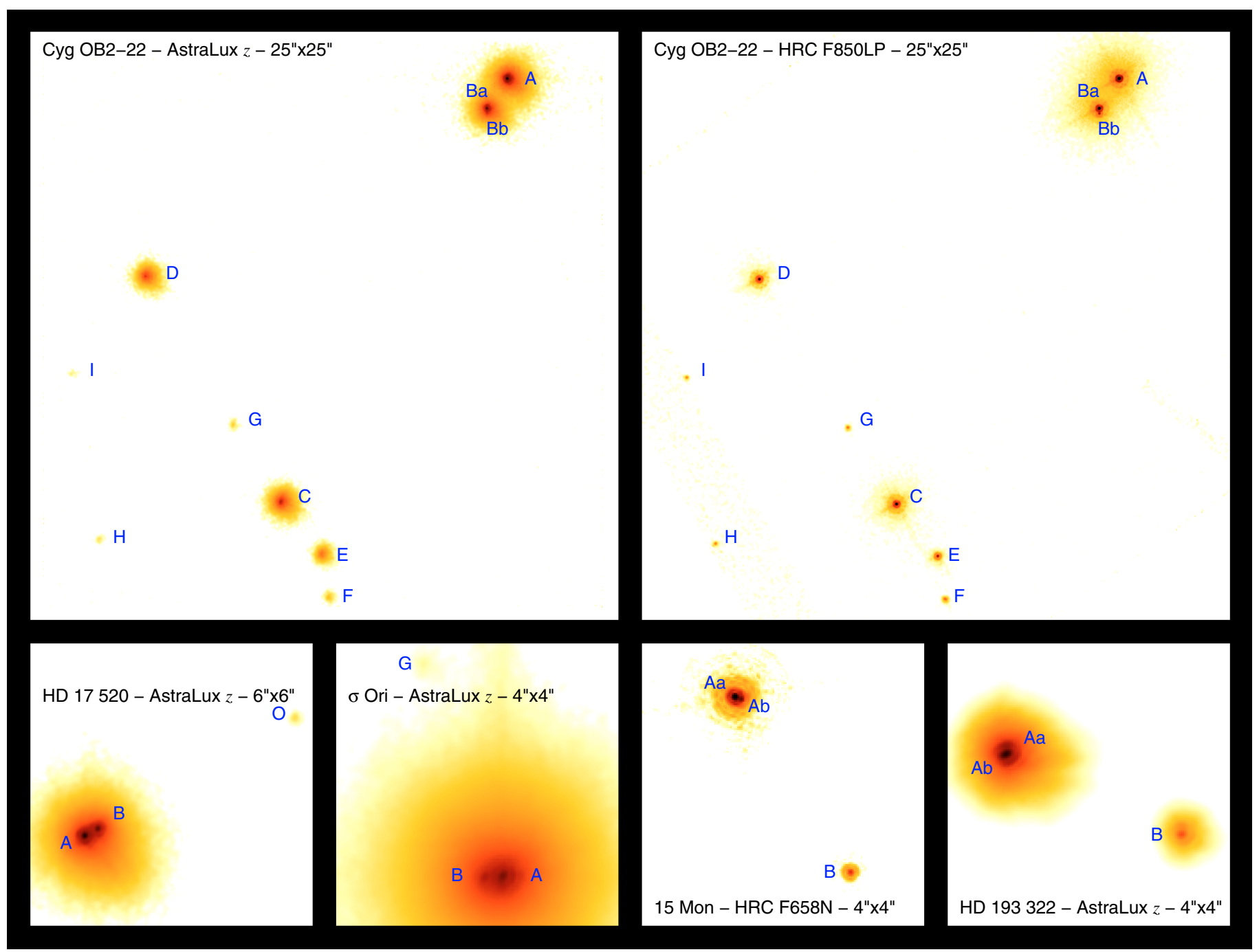

Fig. 1. Sample AstraLux and HRC data used in this paper. The top two panels show the full AstraLux Cyg OB2-22 field (left) and its registered HRC equivalent (right). The bottom four panels show sections of four different systems. In all cases North is up and East is left and a logarithmic stretch is used for the intensity scale. See Sota et al. (in prep.) for an enlargement of the top right panel around $\mathrm{Cyg} \mathrm{OB} 2-22 \mathrm{~A}+\mathrm{Ba}+\mathrm{Bb}$.

Table 1. Systems with a single component in the AstraLux data.

\begin{tabular}{llll}
\hline \hline System name & System name & System name & System name \\
\hline HD 225 160 & HD 18 409 & HD 46573 & MY Ser \\
BD +60 261 & CC Cas & HD 46966 & HD 186 980 \\
HD 12 323 & X Per & V689 Mon & HD 188 209 \\
HD 12 993 & $\xi$ Per & HD 47 417 & 9 Sge \\
V354 Per & HDE 237211 & HD 48 099 & HD 192 639 \\
HD 14 434 & $\alpha$ Cam & HD 52 266 & HD 195 592 \\
HD 14442 & AE Aur & HD 53662 & Cyg OB2-11 \\
HD 14633 & HDE 242908 & HD 53975 & HD 199 579 \\
HD 14947 & BD +34 1058 & HD 54 662 & 68 Cyg \\
HD 15 137 & BD +391328 & HD 54 879 & HD 207 198 \\
BD +60 497 & HD 37 737 & HD 55879 & LZ Cep \\
HD 15 570 & V1382 Ori & HD 57 236 & 19 Cep \\
HD 15642 & HD 41 997 & HD 57 682 & $\lambda$ Cep \\
HD 15629 & HD 42 088 & HD 60 848 & 10 Lac \\
BD +60 513 & HD 45 314 & HD 93 521 & HD 216 532 \\
BD +62 424 & HD 46 149 & $\zeta$ Oph & HD 216 898 \\
HD 16691 & HD 46 223 & Herschel 36 & HD 218915 \\
HD 17603 & HD 46485 & 9 Sgr & BD +60 2522 \\
\hline
\end{tabular}

the search for companions around the earliest Galactic O stars (Maíz Apellániz et al. 2007). The 15 Mon observations were obtained as part of an HST calibration program.

HRC was a $1024 \times 1024$ CCD sensitive in the $2000-10000 \AA$ range with an average pixel scale of $0.027^{\prime \prime} / \mathrm{px}$, hence providing a similar field of view and angular resolution as AstraLux at the same wavelengths where the latter works best. The operative advantages of HRC over AstraLux are its better Strehl ratio, its lack of dependence on atmospheric conditions, and its better contrast because of its single-frame operation. A comparison between the two instruments can be established from the top two panels in Fig. 1. Note how the HRC F850LP halo is significantly less intense than that of the AstraLux $z$ image (both filters are essentially equivalent). The HRC halo in the F850LP images is not an optical effect but is instead caused by scattering in the detector (STScI 2007). At shorter wavelengths the effect disappears, as it can be see in the extremely compact PSF of 15 Mon in one of the lower panels of Fig. 1.

The HRC individual images were processed through the calacs pipeline and combined with multidrizzle to generate geometrically-corrected mosaics. A crowded-field photometry package, JMAPHOT, written by the author for HST data, was used to [a] search for sources in the multidrizzled mosaics, 
Table 2. ACS/HRC measurements of systems with multiple components.

\begin{tabular}{|c|c|c|c|c|c|c|}
\hline System name & Filter & Pair & MJD & $\begin{array}{l}\text { Separation } \\
\left({ }^{\prime \prime}\right)\end{array}$ & $\begin{array}{l}\text { Orientation } \\
\text { (degrees) }\end{array}$ & $\begin{array}{c}\Delta m \\
\text { (mag.) }\end{array}$ \\
\hline \multirow[t]{2}{*}{15 Mon } & F658N & $\mathrm{Aa}-\mathrm{Ab}$ & 53669.96 & $0.089 \pm 0.001$ & $247.90 \pm 0.57$ & $1.62 \pm 0.01$ \\
\hline & & Aa-B & 53669.96 & $2.969 \pm 0.001$ & $213.29 \pm 0.01$ & $3.08 \pm 0.01$ \\
\hline \multirow[t]{9}{*}{ Cyg OB2-22 } & F850LP & A-Ba & 53733.14 & $1.521 \pm 0.001$ & $146.19 \pm 0.03$ & $0.59 \pm 0.01$ \\
\hline & & $\mathrm{Ba}-\mathrm{Bb}$ & 53733.14 & $0.216 \pm 0.001$ & $181.48 \pm 0.25$ & $2.34 \pm 0.01$ \\
\hline & & $\mathrm{A}-\mathrm{C}$ & 53733.14 & $20.404 \pm 0.001$ & $152.37 \pm 0.01$ & $1.15 \pm 0.01$ \\
\hline & & C-D & 53733.14 & $11.191 \pm 0.001$ & $31.37 \pm 0.01$ & $0.57 \pm 0.01$ \\
\hline & & C-E & 53733.14 & $2.821 \pm 0.001$ & $218.41 \pm 0.02$ & $1.73 \pm 0.01$ \\
\hline & & E-F & 53733.14 & $1.850 \pm 0.001$ & $189.82 \pm 0.06$ & $1.89 \pm 0.04$ \\
\hline & & $C-G$ & 53733.14 & $3.853 \pm 0.001$ & $32.41 \pm 0.02$ & $4.02 \pm 0.02$ \\
\hline & & $\mathrm{C}-\mathrm{H}$ & 53733.14 & $7.864 \pm 0.001$ & $102.32 \pm 0.01$ & $4.36 \pm 0.06$ \\
\hline & & C-I & 53733.14 & $10.404 \pm 0.001$ & $58.88 \pm 0.01$ & $4.56 \pm 0.05$ \\
\hline \multirow{4}{*}{ Cyg OB2-7 } & F850LP & A-B & 53736.28 & $18.604 \pm 0.001$ & $104.70 \pm 0.01$ & $2.05 \pm 0.01$ \\
\hline & & $\mathrm{A}-\mathrm{C}$ & 53736.28 & $15.682 \pm 0.001$ & $106.50 \pm 0.01$ & $5.80 \pm 0.02$ \\
\hline & & A-D & 53736.28 & $20.399 \pm 0.001$ & $79.59 \pm 0.01$ & $5.54 \pm 0.01$ \\
\hline & & A-E & 53736.28 & $24.987 \pm 0.004$ & $104.18 \pm 0.01$ & $8.19 \pm 0.15$ \\
\hline
\end{tabular}

and [b] extract the photometry from the individual exposures. The observed photometry was corrected for charge transfer inefficiency effects (STScI 2007). The same sources found in the AstraLux images were detected in the HRC data plus another object (Cyg OB2-7 E) that fell just outside the AstraLux field and just inside the HRC field. JMAPHOT uses the PSFs and geometric distortions of Anderson \& King (2004) for the HRC, which provide excellent photometric and astrometric accuracies. The results for the three HRC fields are shown in Table 2.

Note that for the two Cyg OB2 fields only the F850LP data will be used in this paper. We plan to use the information in the rest of the observed filters in a future work.

\section{Individual systems}

In this section I analyze the systems where there is new significant information compared to the literature. This could be because [a] new components are detected, [b] previously detected components are not seen, [c] the photometry or the astrometry are discrepant with the literature values, or [d] a relative proper motion is detected.

\subsection{HD 5005}

The measured $\Delta z$ values appear to be inconsistent with the Tycho-2 and 2MASS results (all four components are O stars with similar extinctions, see Sota et al. in prep., so they should have similar colors). For Tycho-2, a possible explanation is that $\approx 0.3$ mag from A has been incorrectly assigned to B, hence decreasing the $\Delta m$ between $\mathrm{A}$ and the other three components. In the case of 2MASS, B is not detected and its flux appears to have been distributed between $\mathrm{A}$ and $\mathrm{C}$. The results of Sota et al. (in prep.) for the flux ratios between components in the $B$ band from long-slit spectroscopy agree with the $\Delta z$ values within less than $0.2 \mathrm{mag}$, again indicating a problem with the Tycho- 2 photometry.

The 2MASS and Tycho-2 astrometry for HD 5005 appear to be relatively poor, with offsets of several tenths of an arcsecond.

\section{2. $H D 8768$}

The $H$ and $J$ 2MASS photometry for the $\mathrm{C}$ component are only upper detection limits, probably because of contamination from the unresolved A-B pair. Also, the $\Delta K_{2 \mathrm{M}}$ for A-C is likely overestimated because the flux from B is included in A.

\section{3. $B D+60499$}

The 2MASS position for B is offset by several arcseconds (as can be seen by overplotting the point source catalog on top of e.g. the $J$ image). Also, some of the flux from A is apparently assigned to $\mathrm{B}$, leading to erroneous results for the 2MASS $\Delta m$ values.

\section{4. $H D 16429$}

The Tycho-2 and 2MASS $\Delta m$ values for Aa-B likely refer to $(A a+A b)-B$ but the effect should be small, given the large $\Delta z$ for Aa-Ab. The $\Delta z$ uncertainties are especially large because of a poor PSF fit.

\section{5. $H D 16832$}

A new component $(\mathrm{C})$ is detected.

\section{6. $H D 17505$}

The 2MASS $\Delta m$ values likely refer to $(\mathrm{A}+\mathrm{B})$ rather than to $\mathrm{A}$ alone.

\section{7. $H D 17520$}

A new component $(\mathrm{O})$ is detected (Fig. 1). The 2MASS $\Delta m$ values for A-C likely refer to $(\mathrm{A}+\mathrm{B})-\mathrm{C}$.

\section{8. $B D+60586$}

A new component $(\mathrm{F})$ is detected.

\section{9. $H D 18326$}

A new component $(\mathrm{F})$ is detected. The 2 MASS $\Delta m$ values likely refer to $(\mathrm{A}+\mathrm{B})$ rather than to $\mathrm{A}$ alone. 


\subsection{NSV 1458 - SZ Cam}

This system (WDS $04078+6220$ ) has generated a lot of confusion in the past regarding the use of HD numbers to identify each one of the two stars (Lorenz et al. 1998, Sota et al. in prep.). I observed it with AstraLux using two fields: one that included NSV 1458 (the A component in WDS) plus B, C, and D; and another with the two components of SZ Cam (Ea and Eb in WDS) plus $\mathrm{A}, \mathrm{B}$, and $\mathrm{C}$.

The Ea-Eb pair is one of the three in this paper that I resolve with a separation of less than $0{ }^{\prime} 15$. The uncertainty in $\Delta z$ is much smaller for the Ea-Eb pair than for the other pairs involving Ea because it depends only on the fitting of the stellar cores. For the other pairs (e.g. Ea-A) there is a substantial residual in the fitting due to the assignment of the halo contribution to either $\mathrm{Ea}$ or $\mathrm{Eb}$. On the other hand, it is possible to do simple aperture photometry and obtain a much more reliable value of $\Delta z$ for $(\mathrm{Ea}+\mathrm{Eb})-\mathrm{A}$ of $-0.28 \pm 0.01 \mathrm{mag}$.

$\mathrm{Ea}$ is an eclipsing spectroscopic binary (Lorenz et al. 1998) and I must have caught the system in an eclipse, since the $\Delta z$ value for $(\mathrm{Ea}+\mathrm{Eb})-\mathrm{A}$ is too negative when compared with the Tycho-2 and 2MASS photometry, which do not resolve Ea-Eb. Indeed, the ephemerides of Lorenz et al. (1998) and Gorda et al. (2007) place the observation at a phase distance of just $0.02-$ 0.03 of the secondary eclipse, which is almost as deep as the primary one. The depth of the secondary eclipses given by Lorenz et al. (1998) are $\sim 0.2 \mathrm{mag}$, which is in the range needed to make the result here consistent with the Tycho-2 and 2MASS values. Another point in favor of the system being in an eclipse is that the AstraLux photometry gives $\mathrm{Ea}$ and $\mathrm{Eb}$ as having very similar magnitudes, while the WDS gives a $\Delta m$ for the pair of 0.50 mag. New observations with AstraLux at different epochs should be able to produce a light curve.

At first sight, the astrometry I measure for Ea-Eb indicates a separation somewhat greater than the one predicted by the Gorda et al. (2007) orbit. However, since there is a significant residual in the PSF fitting and the error bars here are relatively large, it is not clear whether the two results are inconsistent or not.

\subsection{1. $A E$ Aur (= HD 34078$)$}

The Ab component is not detected because the measured separation of 0.35 and the expected $\Delta z$ derived from Turner et al. (2008) are likely to be outside the parameter space where AstraLux can identify individual components unless seeing is exceptionally good (see Fig. 2).

\subsection{HDE 242926}

A new component $(\mathrm{B})$ is detected.

\subsection{HDE 242935}

The 2MASS $\Delta m$ values for A-C and A-D likely refer to (A+B)-C and $(\mathrm{A}+\mathrm{B})-\mathrm{D}$, respectively.

\subsection{4. $\theta^{1}$ Ori $(=H D 37020+1+2+3)$}

The 2MASS photometry for the Trapezium stars is inconsistent with the AstraLux and Tycho-2 values and with the NIR speckle holography results of Petr et al. (1998), possibly because of the combined effect of nebular contamination and crowding. I was unable to separate $\mathrm{A}$ or $\mathrm{C}$ into their respective components.

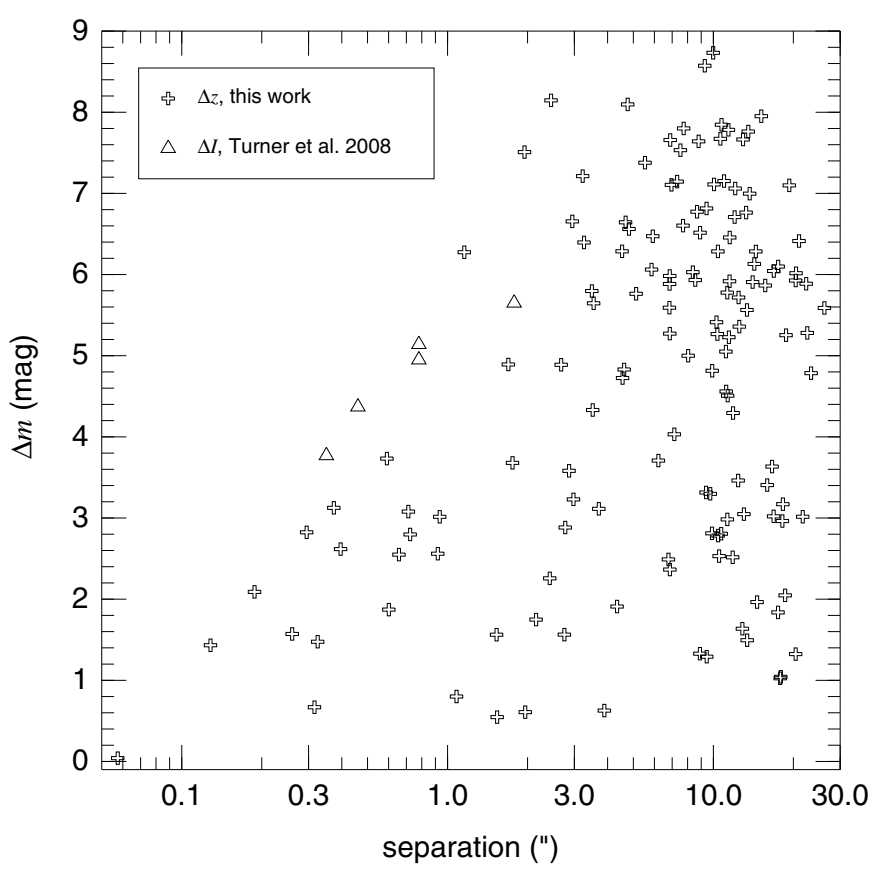

Fig. 2. Separation- $\Delta m$ plot for the pairs detected with AstraLux, where pairs are selected with one of the components being always the brightest star in the field. As a reference, the five pairs with $\Delta m<9.0$ detected by Turner et al. (2008) but not seen in the AstraLux data are also plotted.

In the case of $\mathrm{B}$, I easily resolved $\mathrm{Ba}$ and $\mathrm{Bb}$ but could not detect Bc or Bd (Schertl et al. 2003). The large magnitude difference in $\mathrm{Ba}-\mathrm{Bb}$ confirms the red colors of $\mathrm{Bb}$ (Schertl et al. 2003). Note that the measured $\Delta z$ for $\mathrm{C}$ - $\mathrm{Ba}$ is greater than expected: $\mathrm{Ba}$ is a known eclipsing binary (Stelzer et al. 2005) and I must have observed it during an eclipse. Indeed, the ephemerides of Vitrichenko (2008) provide a phase distance of less than 0.01 between the nearest primary eclipse and the time of observation. Although the $\mathrm{O}-\mathrm{C}$ value could be non-negligible, the eclipse is rather long and its profile near minimum quite flat (Wolf 1994), so the AstraLux observation must have experienced a large magnitude difference with respect to the uneclipsed case. The curves of Wolf (1994) yield a magnitude difference of $\sim 0.5$, which is consistent with the data here.

A new component $(\mathrm{Z})$ not present in either the WDS, Tycho-2, or 2MASS catalogs is detected; note, however, that it had already been identified as TCC 63 by McCaughrean \& Stauffer (1994).

\subsection{5. $\sigma$ Ori $(=H D$ 37468)}

The AstraLux photometric uncertainties are relatively large because of the combination of a poor PSF (see Fig. 1, which shows a PSF elongation along a position angle of $\approx 160^{\circ}$ ) and the proximity of the A and B components. The Tycho-2 and 2MASS $\Delta m$ values likely refer to $(\mathrm{A}+\mathrm{B})$ rather than to $\mathrm{A}$ alone and they may include $\mathrm{G}$ as well, also known as IRS1 (see Caballero 2007, Bouy et al. 2009, and Fig. 1). The separation and orientation for A-B are consistent with the ephemerides of Turner et al. (2008).

\subsection{6. $H D 37366$}

The 2MASS $\Delta m$ values for A-C likely refer to $(\mathrm{A}+\mathrm{B})-\mathrm{C}$. 


\subsection{7. $H D 46202$}

The 2MASS $\Delta m$ are apparently incompatible with the AstraLux results (unless $\mathrm{E}$ is very red), possibly due to crowding. Note that WDS $06321+0458$ A is HD 46180 , located $49^{\prime \prime}$ towards the NW and not included in the AstraLux field of view.

\subsection{8. $H D 46223$}

The B component of Turner et al. (2008) was not detected (see Fig. 2), likely because of a non-optimal seeing of 1'.04.

\subsection{V689 Mon (= HD 47432)}

The B component of Turner et al. (2008) was not detected (see Fig. 2), likely because of a seeing of 1.'08.

\subsection{0. $H D 47032$}

A new component $(\mathrm{B})$ is detected.

\subsection{HD 47129}

The C component of Turner et al. (2008) was not detected (see Fig. 2), likely because of a seeing of 1.'04.

\subsection{Mon (= HD 47839)}

$\mathrm{Aa}-\mathrm{Ab}$ is another one of the closest pairs analyzed in this paper. The $\Delta V_{\text {Ty }}$ value for Aa-B likely refers to $(\mathrm{Aa}+\mathrm{Ab})-\mathrm{B}$.

The Aa-Ab orbit has been followed for some time now (Gies et al. 1997; Mason et al. 2009) and the most recently published orbit (Cvetković et al. 2010) is significantly wider than the previous one of Gies et al. (1997). In this paper I publish two new points, one for October 2005 (HRC, Fig. 1) and another one for January 2008 (AstraLux). The 2005 value is consistent with the Cvetković et al. (2010) orbit but the 2008 value is not, indicating that $\mathrm{Ab}$ is moving faster and in a wider orbit. If one looks at Fig. 1 in Cvetković et al. (2010) it is clear that their last two measurements are also discrepant. The AstraLux results point in the direction of the outer one of those two measurements being closer to the truth than the inner one.

The astrometry for Aa-B and the photometry for the two pairs show a good agreement between HRC and AstraLux (within the expected color term between F658N and z), hence providing a confirmation of the AstraLux calibration.

\subsection{3. $H D 52533$}

The 2MASS $\Delta m$ values for A-E and A-F likely refer to $(\mathrm{A}+\mathrm{B})-\mathrm{E}$ and $(\mathrm{A}+\mathrm{B})-\mathrm{F}$, respectively.

\subsection{4. $H D 193322$}

$\mathrm{Aa}-\mathrm{Ab}$ is the closest pair that I am able to identify in the sample in this paper, with a separation of only $55 \pm 16$ mas. The identification is possible thanks to the existence of the bright B companion, which allows for an accurate PSF modeling (see Fig. 1, where B appears to have good circular symmetry and $\mathrm{Aa}+\mathrm{Ab}$ appear as an elongated single object). The separation and orientation are compatible with the Mason et al. (2009) results. The $\Delta z$, which is rather uncertain, is quite different to the Mason et al. (2009) value for $\Delta m$ but within 2 sigma of the
McKibben et al. (1998) result. Note that the $\Delta V_{\text {Ty }}$ value refers to $(A a+A b)-B$.

\subsection{Cyg OB2-5 (= V279 Cyg $=B D+404220)$}

A new component (D) is detected. The 2MASS $\Delta m$ values for A-C likely refer to $(\mathrm{A}+\mathrm{B})-\mathrm{C}$. Note that the component referred to as D in Kennedy et al. (2010) is B here and in the WDS.

\subsection{Cyg OB2-22}

There are five components detected (Bb, E, F, G, and I) in the AstraLux data that are not present in the WDS or 2MASS (this system is too dim for Tycho-2), for a total of 10 components (Fig. 1). Of the five new components, the last four are detected by Massey \& Thompson (1991) as objects 420, 807, 814, and 426, respectively. The 2 MASS $\Delta m$ values likely refer to $(\mathrm{A}+\mathrm{Ba}+\mathrm{Bb})$ rather than to $\mathrm{A}$ alone. The same ten components are detected with ACS/HRC.

The AstraLux $\Delta z$ values are in general consistent with the HRC F850LP results with two caveats. [1] The AstraLux value for $\mathrm{Ba}-\mathrm{Bb}$ has a very large uncertainty because $\mathrm{Bb}$ is close to the detection limit for its $\Delta z$ and separation. On the other hand, HRC cleanly separates the two. [2] C appears to be too dim in the AstraLux photometry with respect to HRC by $0.05-0.10$ magnitudes. One possibility is that $\mathrm{C}$ is variable.

With the exception of $\mathrm{Ba}-\mathrm{Bb}$, the AstraLux separations and orientations for all pairs are consistent with the HRC results, thus validating the Trapezium-based AstraLux astrometric calibration used in this paper (no significant proper motions are expected in the $\approx 2$ years that lapsed between the HRC and AstraLux observations). The $\mathrm{Ba}-\mathrm{Bb}$ separation may have increased while maintaining a near-constant orientation, but such a motion is only a $2 \sigma$ detection in a close system with a relatively large $\Delta z$, so it may not be real.

\subsection{Cyg OB2-7}

A new component $(\mathrm{C})$ is detected in both the AstraLux and the HRC data and another one (E) in the HRC images (E is just outside the AstraLux field of view). The astrometry for AB, AC, and AD show a good agreement between HRC and AstraLux, hence providing another confirmation of the AstraLux calibration. The photometry also shows a good agreement for $\mathrm{AB}$ and $\mathrm{AC}$ but not for $\mathrm{AD}$. A possible explanation is that $\mathrm{D}$ is variable.

\subsection{HD 206267}

The Tycho-2 $\Delta m$ values appear to be incompatible with the AstraLux $\Delta z$, the 2MASS $\Delta m$, and the published $B V$ photometry (Nicolet 1978). The B component of Turner et al. (2008) is undetected because of the very bad seeing (1'.85, see Fig. 2).

\subsection{9. $\mathrm{DH}$ Cep (= HD 215835)}

A new component (B) is detected.

\subsection{HD 218195}

A new component $(\mathrm{C})$ is detected. The 2MASS $\Delta m$ values for A-D likely refer to $(\mathrm{A}+\mathrm{B}+\mathrm{C})-\mathrm{D}$. 


\section{Discussion}

\subsection{Overall strategy}

This paper is the first step of a long-term project to study the multiplicity of massive stars. The project will include further high-angular resolution imaging, multiple-epoch $R \sim$ 40000 optical spectroscopy (Gamen et al. 2008), a recalibration of the intrinsic colors for hot stars and the extinction law (Maíz Apellániz \& Sota 2008), and a combination of spectroscopic and trigonometric parallaxes (Maíz Apellániz et al. 2008). Regarding high-resolution imaging, the goal is to eventually observe 500 massive stars with AstraLux and, if a similar instrument becomes available in the Southern hemisphere, to observe another $\sim 500$ stars from there, in all cases using the Galactic O-Star Catalog (Maíz Apellániz et al. 2004; Sota et al. 2008) to select the sample.

Literature results will also be included in our final analysis. In that respect, it is useful to compare the sample size and AstraLux capabilities with previous large-scale surveys. Mason et al. (1998, 2009) observed 400 O and WR stars with speckle interferometry, which has the advantage of being able to reach down to lower separations $\left(0{ }^{\prime} 03\right)$ but the disadvantages of lower maximum separations $\left(1^{\prime \prime} .5\right)$ and dynamic range $(\Delta m \sim 3)$. Nelan et al. (2004) used the Fine Guidance Sensor (FGS) 1r aboard HST as an interferometer to observe $23 \mathrm{OB}$ stars in the Carina Nebula. FGS can reach to even smaller separations than speckle interferometry but reaches out only to $\sim 0$.'5. Turner et al. (2008) observed a sample similar in size to the one here with $I$-band adaptive optics. Their instrument explores a similar region of the separation- $\Delta m$ plane as AstraLux, with a slight advantage in $\Delta m$ ( 1.5 mag at separations of 3-6", see Fig. 2 where 18 objects with $\Delta m \sim 10$ detected by Turner et al. 2008 lie outside the plotted range) and disadvantages in separation range ( 6 " instead of $12-18^{\prime \prime}$ ) and $\Delta m$ precision.

\subsection{Completeness}

Figure 2 shows the separation and $\Delta z$ of all of our detected pairs, where values are measured from the brightest star, not for the pairs listed in Table 3 (the same criterion applies to subsequent figures). There is an obvious detection limit that runs from $\approx 0$ !' 1 for small $\Delta z$ to $\approx 2^{\prime \prime}$ for $\Delta z=8 \mathrm{mag}^{2}$. The location of the detection limit is not the same for all of the observations because it is seeing-dependent. A consequence of this dependence is that one of the five AstraLux non-detections on Fig. 2 (HD 206 267) is surrounded by AstraLux detections due the very bad seeing of that particular AstraLux observation. The AstraLux detections also appear to become affected by incompleteness at large distances for $\delta z>8$. From Fig. 2 it can be estimated that if the $\Delta m$ distribution is independent of separation, then $\sim 30$ stars with separations between 0.1 and $2^{\prime \prime}$ and $\Delta z<8$ are undetected by AstraLux.

\subsection{Bound or not?}

A basic question that needs to be answered is how many of the observed pairs are [a] real, bound pairs and how many are either [b] stars located in the same cluster but not bound to the central massive stars or [c] chance alignments from stars located along

\footnotetext{
2 Note that the point near the lower left corner, which corresponds to HD $193322 \mathrm{Aa}-\mathrm{Ab}$, is a special case that is detected because the existence of nearby, bright B allows for a better-than-normal PSF modeling, see above.
}

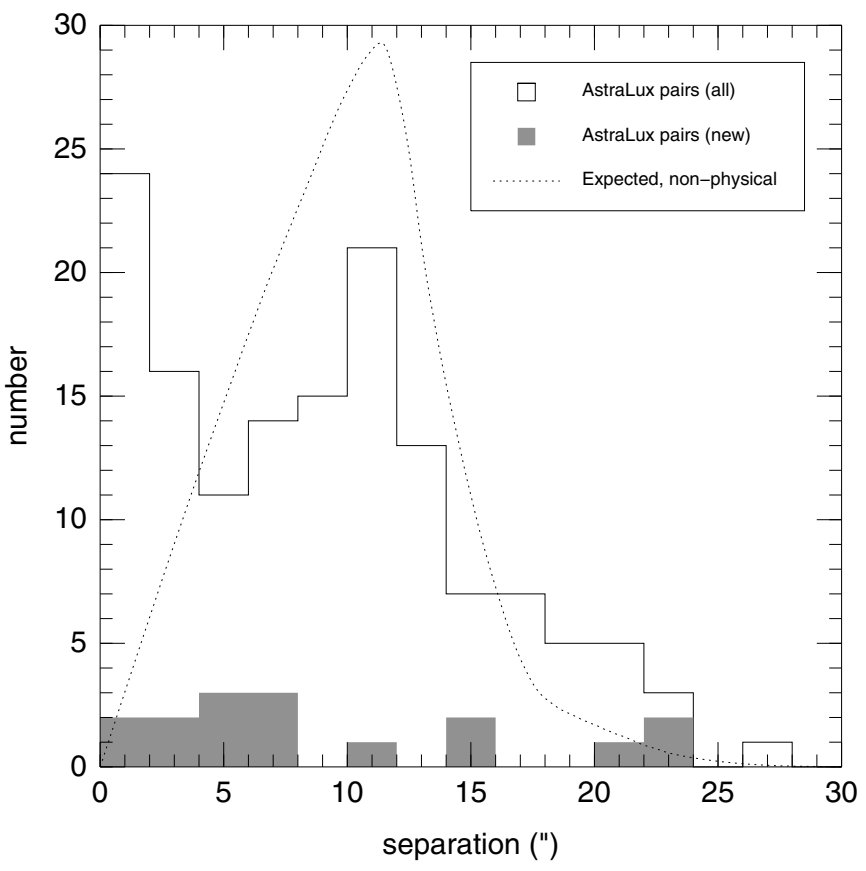

Fig. 3. Separation histogram for all and for new pairs detected with AstraLux. The dotted line indicates the expected shape for a population of non-physical pairs uniformly distributed in the plane of the sky. As in the previous figure, pairs are selected with one of the components being always the brightest star in the field. The new pairs with large separations belong to systems with many components; if measured from the nearest companion, their separations would be considerably smaller.

the Galactic line of sight (foreground or background). To solve this issue with certainty, one would need to measure the proper motions and velocities of each component, a task that is beyond the scope of this first paper (but that will be eventually tackled within the GOSSS project). Here I attempt a statistical approach with the help of Fig. 3, which shows the separation histogram for the AstraLux-detected pairs. It also shows the expected nonnormalized distribution for pairs built by associating the central massive star with a population of stars uniformly distributed over the field of view. Such an expected distribution initially increases linearly with separation and then turns around where the detector edge is reached. It was built taking into account the distribution of the central stars on the AstraLux $\mathrm{CCD}^{3}$.

A comparison between the observed separation of the AstraLux pairs and the expected distribution described above shows an important difference: a strong peak is present at small separations. Furthermore, as previously discussed, the first bin that defines this peak $\left(0^{\prime \prime}-2^{\prime \prime}\right.$, in practical terms the lower limit is something like 0.1 ) should be strongly affected by incompleteness so its real height should be roughly double what is shown in Fig. 3. The conclusion is that this peak is formed by real, bound pairs and not by cluster or Galactic chance alignments.

On the other hand, for separations beyond 6 " the observed distribution is relatively well-approximated by the expected distribution formed by a uniform population. There may be an excess of objects with large separations $\left(>16^{\prime \prime}\right)$ but this can be simply a selection effect due to the specific positioning of the

\footnotetext{
3 As previously mentioned, for some targets the brightest star was located off-center in order to observe previously-known components. For some cases with a large separation the pair was aligned close to the NW-SE or NE-SW directions.
} 
targets when the existence of a component was known in advance. Note, however, that the similarity of the two distributions does not allow us to distinguish between options [b] and [c] above or even a specific case of [a], a population of bound pairs with large separations spread over a broad range. In order to differentiate between the three we can take advantage of the fact that for separations beyond 8 " one would expect 2MASS detections to be relatively complete (i.e. unaffected by the presence of a bright nearby star) for $\Delta m<9$. This can be seen from the fact that in the separation range $8^{\prime \prime}-14^{\prime \prime}$ forty-six ${ }^{4}$ out of fortynine the AstraLux detections are also in 2MASS (two more are in the WDS and the last one is a new detection) and can also be deduced from Fig. 6 in Turner et al. (2008). Therefore, we can use 2MASS to estimate the fractions of the pairs in the peak around $11^{\prime \prime}$ of separation that are either bound systems, cluster members, or unrelated Galactic alignments.

I start by defining three separation ranges for 2MASS: [1] the stellar system $\left(8^{\prime \prime}-14^{\prime \prime}\right)$, [2] the cluster $\left(1^{\prime}-4^{\prime}\right)$, and [3] the Galactic fore/background $\left(30^{\prime}-40^{\prime}\right)^{5}$. As previously mentioned, AstraLux detects 46 objects (in 33 fields) in the stellar-sytem range. To that number I apply a geometric correction that accounts for the incomplete angle coverage to determine that AstraLux would detect $\sim 61$ sources between $8^{\prime \prime}$ and $14^{\prime \prime}$ if it had covered that separation range in full. On the other hand, there are 106 2MASS detections (in at least one band) in that range for the same fields, indicating the existence of a detection limit in magnitude for Astralux with respect to the 2MASS data (i.e. 2MASS is deeper for those separations). I then [a] calculate the fraction of AstraLux-detected stars for each of the three 2MASS bands ( $J, H$, and $K_{\mathrm{S}}$ ); [b] convert the result to 2MASS magnitude limits for the AstraLux detections; and [c] obtain the 2MASS stellar density above those magnitude limits at the other two separation ranges (cluster and Galactic) centered on each of the 33 AstraLux fields. From those values and assuming a Poisson distribution, I obtain the probability that the observed AstraLux pair or pairs in the stellar system range belongs to either [a], [b], or [c] ${ }^{6}$. I then sum over the 33 AstraLux fields and I obtain the percentage of pairs that falls in each category. Since the process is repeated for the three 2MASS bands, I derive a mean value and a dispersion for each of the percentages.

The result of the above procedure is that for separations of $8^{\prime \prime}-14^{\prime \prime}, 78 \pm 6 \%$ of the pairs are bound, $10 \pm 2 \%$ are chance alignments with a cluster member, and $12 \pm 4 \%$ are chance alignments with a non-cluster (Galactic fore/background) member. These numbers indicate a rather large proportion of bound pairs in the AstraLux sample. The fraction of bound stars is slightly greater than the values reported by Turner et al. (2008) but it also represents a different range in separation and $\Delta m$ (more specifically, their sample includes many stars with $\Delta m \sim 10 \mathrm{mag}$ ). It is worth mentioning that there are no dense clusters with $\mathrm{O}$ stars above a declination of $-25^{\circ}$ (NGC 3603, Trumpler 14, and NGC 6231 are all further south, for example); in those cases one would expect a higher fraction for chance alignments with cluster members (Maíz Apellániz 2008). Also, it should be remembered that

\footnotetext{
${ }^{4}$ In thirty-three different fields, some with two or three pairs.

5 The limits for [2] and [3] are realistic but somewhat arbitrary and the method here assumes that the surface density for cluster and Galactic members does not vary between the center and the maximum separation in each case, making this estimate a rather unsophisticated one. Nevertheless, the results below were found to be robust with respect to small changes in the limits.

6 The probability for [a] and [c] is always found to be non-zero. This is not always true for $[\mathrm{b}]$, since some $\mathrm{O}$ stars do not belong to a cluster.
}

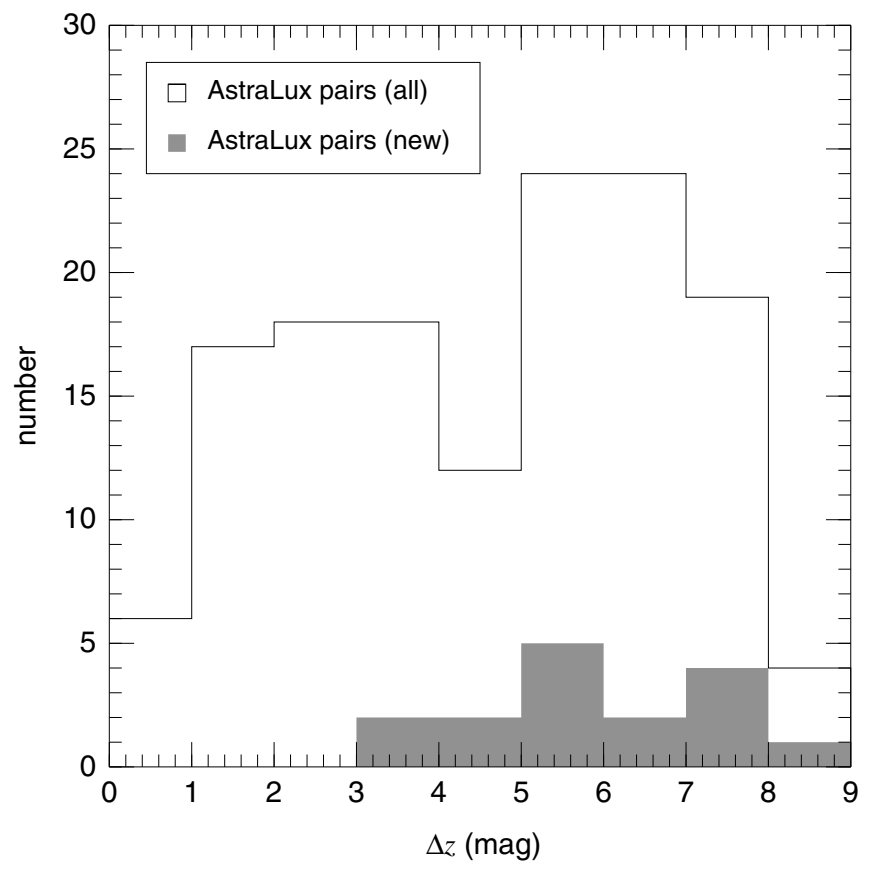

Fig. 4. $\Delta m$ histogram for all and for new pairs detected with AstraLux. As in the two previous figures, pairs are selected with one of the components being always the brightest star in the field.

these values are preliminary pending a larger sample and proper motion and radial velocity studies.

\subsection{The multiplicity fraction}

The quest for the multiplicity fraction is the ultimate goal of a work like this. Unfortunately, at this stage there are many limitations for the numbers that can be provided based on the current data.

1. The most important one is the contribution from short-period spectroscopic binaries (SBs), many of which have separations well beyond AstraLux capabilities. Mason et al. (1998) already found multiplicity fractions of 30-50\% among SBs and recent works have found many new discoveries. Indeed, within GOSSS we have found several tens of new SBs (Barbá et al., in preparation), so the SB fraction should be even higher.

2. $\Delta m \sim 8-9$ (the limit here) is not the expected limit for bound companions (Turner et al. 2008; Maíz Apellániz et al. 2010). Also, a look at Fig. 4 shows that new discoveries tend to be produced at large $\Delta m$, indicating that widening the search in $\Delta m$ will also likely increase the multiplicity fraction.

3. As previously mentioned, our numbers for separations in the $0 . ' 1-2^{\prime \prime}$ range must depend on completeness corrections. Also note in Fig. 3 that several new AstraLux pairs are found at low separations.

4. Finally, there is the issue of the outer limit for bound pairs. $10^{\prime \prime}$ at the typical distances of 1-2 kpc for the objects in our sample is 10000-20000 AU, which is close to the limit one would expect for bound pairs involving a massive $\operatorname{star}^{7}$

\footnotetext{
7 Not only for dynamical reasons but also because for larger separations the system would have time for only one or a few orbits before the central star explodes, hence questioning whether we should consider such a system a binary or not.
} 
Therefore, we can consider that the completeness correction due to possible large-separation missed systems is likely small.

With the above caveats in mind I derive a number for the multiplicity fraction for objects with separations of $0 .{ }^{\prime} 1-14^{\prime \prime}$ with $\Delta z<8$. The rough percentage (66 fields with two or more components out of a total of 138) yields $48 \pm 4 \%$. That value needs to be corrected for two reasons: First, we should consider that $\approx 22 \%$ ( $\sim 20$ cases) of the large separation pairs are not bound ${ }^{8}$. Second, we have estimated that there are $\sim 30$ undetected pairs in the 0 ! $^{\prime} 1-2^{\prime \prime}$ range. Since those two numbers are similar and they work in opposite directions, I conclude that approximately $50 \%$ of the objects in the sample are multiple within the range of separations and $\Delta z$ above, with a more precise number depending on further analysis. Of course, the total multiplicity fraction for massive stars should be much higher.

Acknowledgements. I would like to thank Felix Hormuth for helping with the use of the AstraLux instrument and pipeline; Brian D. Mason for providing corrections for some of the data in Table 2; Alfredo Sota for obtaining complementary information with long-slit optical spectroscopy; the IAA Director, Paco T.D.W. Prada, for granting the time for this project; and Nolan R. Walborn and the referee, Doug Gies, for useful comments that improved the manuscript. This research was conducted using observing time provided by the IAA-CSIC Director Guaranteed Time for the Calar Alto Observatory. Support for this work was provided by [a] the Spanish Government Ministerio de Ciencia e Innovación through grants AYA2007-64052, the Ramón y Cajal Fellowship program, and FEDER funds; [b] the Junta de Andalucía grant P08-TIC-4075; and [c] NASA through grant GO-10602 from the Space Telescope Science Institute, which is operated by the Association of Universities for Research in Astronomy Inc., under NASA contract NAS 5-26555. This research has made use of [a] Aladin (Bonnarel et al. 2000); [b] the SIMBAD database, operated at CDS, Strasbourg, France; [c] data products from the Two Micron All Sky Survey, which is a joint project of the University of Massachusetts and the Infrared Processing and Analysis Center/California Institute of Technology, funded by the National Aeronautics and Space Administration and the National Science Foundation; and [d] the Tycho-2 catalog (Høg et al. 2000).

\section{References}

Anderson, J., \& King, I. R. 2004, ACS Instrument Science Report 2004-15 (STScI: Baltimore)

Bonnarel, F., Fernique, P., Bienaymé, O., et al. 2000, A\&AS, 143, 33
Bouy, H., Huélamo, N., Martín, E. L., et al. 2009, A\&A, 493, 931 Caballero, J. A. 2007, A\&A, 466, 917

Cvetković, Z., Vince, I., \& Ninković, S. 2010, New Astron., 15, 302 Fruchter, A. S., \& Hook, R. N. 2002, PASP, 114, 144

Gamen, R., Barbá, R. H., Morrell, N. I., Arias, J., \& Maíz Apellániz, J. 2008, in Rev. Mex. Astron. Astrofís. Conf. Ser., 33, 54

Gies, D. R., Mason, B. D., Bagnuolo, Jr., W. G., et al. 1997, ApJ, 475, L49

Gorda, S. Y., Balega, Y. Y., Pluzhnik, E. A., \& Shkhagosheva, Z. U. 2007, Astrophysical Bulletin, 62, 352

Høg, E., Fabricius, C., Makarov, V. V., et al. 2000, A\&A, 355, L27

Hormuth, F., Brandner, W., Hippler, S., \& Henning, T. 2008, J. Phys. Conf. Ser., 131, 012051

Kennedy, M., Dougherty, S. M., Fink, A., \& Williams, P. M. 2010, ApJ, 709, 632

Lorenz, R., Mayer, P., \& Drechsel, H. 1998, A\&A, 332, 909

Maíz Apellániz, J. 2008, ApJ, 677, 1278

Maíz Apellániz, J., \& Sota, A. 2008, in Rev. Mex. Astron. Astrofís. 33, 44

Maíz Apellániz, J., Walborn, N. R., Galué, H. Á., \& Wei, L. H. 2004, ApJS, 151, 103

Maíz Apellániz, J., Walborn, N. R., Morrell, N. I., Niemelä, V. S., \& Nelan, E. P. 2007, ApJ, 660, 1480

Maíz Apellániz, J., Alfaro, E. J., \& Sota, A. 2008 [arXiv:0804 . 2553]

Maíz Apellániz, J., Walborn, N. R., Morrell, N. I., Nelan, E. P., \& Niemelä, V. S. 2010, in Joint Discussion 13, Eta Carinae in the Context of the Most Massive Stars, Highlights of Astronomy, 14 XXVIth IAU General Assembly

Mason, B. D., Gies, D. R., Hartkopf, W. I., et al. 1998, AJ, 115, 821

Mason, B. D., Wycoff, G. L., Hartkopf, W. I., Douglass, G. G., \& Worley, C. E. 2001, AJ, 122, 3466

Mason, B. D., Hartkopf, W. I., Gies, D. R., Henry, T. J., \& Helsel, J. W. 2009 , AJ, 137, 3358

Massey, P., \& Thompson, A. B. 1991, AJ, 101, 1408

McCaughrean, M. J., \& Stauffer, J. R. 1994, AJ, 108, 1382

McKibben, W. P., Bagnuolo, Jr., W. G., Gies, D. R., et al. 1998, PASP, 110, 900 Moffat, A. F. J. 1969, A\&A, 3, 455

Nelan, E. P., Walborn, N. R., Wallace, D. J., et al. 2004, AJ, 128, 323

Nicolet, B. 1978, A\&AS, 34, 1

Öpik, E. J. 1924, Tartu Obs. Publ., 25

Petr, M. G., Coude Du Foresto, V., Beckwith, S. V. W., Richichi, A., \& McCaughrean, M. J. 1998, ApJ, 500, 825

Schertl, D., Balega, Y. Y., Preibisch, T., \& Weigelt, G. 2003, A\&A, 402, 267

Skrutskie, M. F., Cutri, R. M., Stiening, R., et al. 2006, AJ, 131, 1163

Sota, A., Maíz Apellániz, J., Walborn, N. R., \& Shida, R. Y. 2008, in Rev. Mex. Astron. Astrofís. Conf. Ser., 33, 56

Stelzer, B., Flaccomio, E., Montmerle, T., et al. 2005, ApJS, 160, 557

STScI. 2007, ACS Instrument Handbook, ed. F. R. Boffi, et al.

Turner, N. H., ten Brummelaar, T. A., Roberts, L. C., et al. 2008, AJ, 136, 554

Vitrichenko, É. A. 2008, Astrophysics, 51, 424

Walborn, N. R., Howarth, I. D., Lennon, D. J., et al. 2002, AJ, 123, 2754

Walborn, N. R., Sota, A., Maíz Apellániz, J., et al. 2010, ApJ, 711, L143

Wolf, G. W. 1994, Experimental Astronomy, 5, 61

\footnotetext{
${ }^{8}$ Some of those pairs will be in systems with two or more pairs so the reduction is not as drastic as if they were all in single-pair systems.
} 
Table 3. AstraLux measurements of systems with multiple components.

\begin{tabular}{|c|c|c|c|c|c|c|c|c|c|c|}
\hline System name & WDS & Pair & MJD & $\begin{array}{c}\text { Separation } \\
\left({ }^{\prime \prime}\right)\end{array}$ & $\begin{array}{c}\text { Orientation } \\
\text { (degrees) }\end{array}$ & $\begin{array}{c}\text { pair } \\
\text { in WDS? }\end{array}$ & $\begin{array}{c}\Delta V_{\mathrm{Ty}} \\
\text { (mag.) }\end{array}$ & $\begin{array}{c}\Delta z \\
\text { (mag.) }\end{array}$ & $\begin{array}{l}\Delta H_{2 \mathrm{M}} \\
\text { (mag.) }\end{array}$ & $\begin{array}{l}\Delta K_{2 \mathrm{M}} \\
\text { (mag.) }\end{array}$ \\
\hline HD 225146 & $00040+6106$ & A-B & 54483.76 & $8.802 \pm 0.016$ & $75.16 \pm 0.09$ & $\mathrm{n}$ & & $7.64 \pm 0.06$ & 5.70 & 5.63 \\
\hline HD 108 & $00061+6341$ & $A-C$ & 54482.78 & $13.281 \pm 0.018$ & $227.69 \pm 0.07$ & $\mathrm{n}$ & $\ldots$ & $6.76 \pm 0.07$ & 6.27 & 6.22 \\
\hline HD 1337 & $00177+5126$ & $\mathrm{~A}-\mathrm{C}$ & 54482.76 & $12.933 \pm 0.019$ & $103.68 \pm 0.08$ & $\mathrm{n}$ & $\ldots$ & $7.66 \pm 0.05$ & 6.14 & 6.12 \\
\hline \multirow[t]{3}{*}{ HD 5005} & $00528+5638$ & $A-B$ & 54416.94 & $1.529 \pm 0.016$ & $81.62 \pm 0.48$ & $\mathrm{y}$ & 0.75 & $1.56 \pm 0.03$ & & \\
\hline & & $\mathrm{A}-\mathrm{C}$ & 54416.94 & $3.889 \pm 0.017$ & $133.12 \pm 0.42$ & $\mathrm{y}$ & 0.31 & $0.63 \pm 0.02$ & 0.13 & 0.26 \\
\hline & & A-D & 54416.94 & $8.902 \pm 0.022$ & $193.53 \pm 0.40$ & $\mathrm{y}$ & 1.07 & $1.33 \pm 0.02$ & 1.74 & 1.77 \\
\hline \multirow[t]{2}{*}{ HD 8768} & $01281+6317$ & A-B & 54761.13 & $0.656 \pm 0.016$ & $27.54 \pm 0.38$ & $\mathrm{y}$ & $\ldots$ & $2.55 \pm 0.02$ & & \\
\hline & & $\mathrm{A}-\mathrm{C}$ & 54761.13 & $5.918 \pm 0.016$ & $24.28 \pm 0.13$ & $\mathrm{n}$ & $\ldots$ & $6.47 \pm 0.03$ & ${ }^{a} 2.69$ & 5.66 \\
\hline HD 10125 & $01409+6410$ & A-B & 54482.82 & $0.715 \pm 0.016$ & $232.52 \pm 0.38$ & $\mathrm{y}$ & $\ldots$ & $3.08 \pm 0.04$ & & \\
\hline HD 13022 & $02095+5847$ & A-B & 54483.78 & $13.382 \pm 0.016$ & $129.88 \pm 0.07$ & $\mathrm{n}$ & $\ldots$ & $5.56 \pm 0.14$ & 4.08 & 3.93 \\
\hline HD 13268 & $02115+5610$ & A-B & 54761.14 & $10.734 \pm 0.017$ & $200.78 \pm 0.08$ & $\mathrm{n}$ & $\ldots$ & $7.85 \pm 0.06$ & 7.06 & 6.95 \\
\hline \multirow[t]{2}{*}{$\mathrm{BD}+60499$} & $02323+6133$ & A-B & 54483.82 & $4.617 \pm 0.016$ & $226.30 \pm 0.15$ & $\mathrm{n}$ & $\ldots$ & $4.83 \pm 0.02$ & ${ }^{a} 0.90$ & ${ }^{a} 0.89$ \\
\hline & & $\mathrm{A}-\mathrm{C}$ & 54483.82 & $14.054 \pm 0.017$ & $119.60 \pm 0.06$ & $\mathrm{n}$ & $\ldots$ & $5.91 \pm 0.14$ & 4.39 & 4.30 \\
\hline \multirow[t]{2}{*}{ BD +60 501} & $02326+6128$ & A-B & 54483.83 & $12.024 \pm 0.016$ & $57.19 \pm 0.07$ & $\mathrm{n}$ & $\ldots$ & $6.71 \pm 0.05$ & 5.62 & 5.45 \\
\hline & & A-C & 54483.83 & $7.517 \pm 0.017$ & $296.05 \pm 0.11$ & $\mathrm{n}$ & & $7.53 \pm 0.06$ & 5.36 & 5.28 \\
\hline \multirow[t]{3}{*}{ HD 15558} & $02327+6127$ & A-B & 54483.84 & $9.883 \pm 0.016$ & $96.79 \pm 0.08$ & $\mathrm{y}$ & 2.10 & $2.81 \pm 0.05$ & 2.77 & 2.76 \\
\hline & & $A-F$ & 54483.84 & $4.536 \pm 0.016$ & $263.27 \pm 0.15$ & $\mathrm{y}$ & $\ldots$ & $6.29 \pm 0.09$ & & \\
\hline & & A-I & 54483.84 & $10.626 \pm 0.016$ & $5 \pm 0.08$ & $\mathrm{n}$ & $\ldots$ & $7.67 \pm 0.05$ & ${ }^{a} 5.70$ & 6.08 \\
\hline \multirow[t]{3}{*}{ HD 16429} & $02407+6117$ & $\mathrm{Aa}-\mathrm{Ab}$ & 54483.87 & $0.295 \pm 0.016$ & $91.73 \pm 0.52$ & $\mathrm{y}$ & & $2.82 \pm 0.61$ & & \\
\hline & & Aa-B & 54483.87 & $6.777 \pm 0.016$ & $3 \pm 0.11$ & $\mathrm{y}$ & 1.97 & $2.49 \pm 0.22$ & 2.37 & 2.44 \\
\hline & & Aa-D & 54483.87 & $2.947 \pm 0.104$ & $112.54 \pm 2.33$ & $\mathrm{y}$ & $\ldots$ & $6.66 \pm 1.33$ & & \\
\hline \multirow[t]{2}{*}{ HD 16832} & $02442+5639$ & A-B & 54483.81 & $12.085 \pm 0.016$ & \pm 0.07 & $\mathrm{n}$ & $\ldots$ & $7.06 \pm 0.04$ & 5.74 & 5.55 \\
\hline & & $\mathrm{A}-\mathrm{C}$ & 54483.81 & $4.761 \pm 0.017$ & $335.52 \pm 0.15$ & $\mathrm{n}$ & & $8.10 \pm 0.08$ & $\ldots$ & \\
\hline \multirow[t]{5}{*}{ HD 17505} & $02511+6025$ & A-B & 54483.90 & $2.153 \pm 0.016$ & $92.67 \pm 0.24$ & $\mathrm{y}$ & 1.70 & $1.75 \pm 0.03$ & $\ldots$ & \\
\hline & & A-I & 54483.90 & $4.676 \pm 0.016$ & $144.09 \pm 0.15$ & $\mathrm{y}$ & $\ldots$ & $6.64 \pm 0.02$ & & \\
\hline & & A-J & 54483.90 & $8.912 \pm 0.016$ & $96.13 \pm 0.09$ & $\mathrm{n}$ & $\ldots$ & $6.52 \pm 0.02$ & ${ }^{a} 4.88$ & ${ }^{a} 5.73$ \\
\hline & & A-K & 54483.90 & $13.698 \pm 0.017$ & $313.82 \pm 0.06$ & $\mathrm{n}$ & $\ldots$ & $7.00 \pm 0.06$ & 6.17 & 6.00 \\
\hline & & A-L & 54483.90 & $13.531 \pm 0.017$ & $224.52 \pm 0.07$ & $\mathrm{n}$ & $\ldots$ & $7.76 \pm 0.05$ & 6.72 & 6.54 \\
\hline \multirow[t]{3}{*}{ HD 17520} & $02512+6023$ & A-B & 54483.88 & $0.316 \pm 0.016$ & $297.38 \pm 0.61$ & $\mathrm{y}$ & $\ldots$ & $0.67 \pm 0.07$ & & \\
\hline & & $\mathrm{A}-\mathrm{C}$ & 54483.88 & $10.702 \pm 0.016$ & $20.42 \pm 0.08$ & $\mathrm{y}$ & $\ldots$ & $2.81 \pm 0.05$ & 3.15 & 3.24 \\
\hline & & $\mathrm{A}-\mathrm{O}$ & 54483.88 & $5.122 \pm 0.016$ & $1 \pm 0.14$ & $\mathrm{n}$ & $\ldots$ & $5.76 \pm 0.06$ & & \\
\hline \multirow{7}{*}{ BD +60 586} & $02542+6039$ & $A-B$ & 54483.82 & $7.131 \pm 0.016$ & $233.50 \pm 0.11$ & $\mathrm{y}$ & $\ldots$ & $4.03 \pm 0.14$ & 3.58 & 3.48 \\
\hline & & $\mathrm{A}-\mathrm{C}$ & 54483.82 & $15.946 \pm 0.017$ & \pm 0.06 & $\mathrm{y}$ & $\ldots$ & $3.41 \pm 0.13$ & 3.12 & 3.07 \\
\hline & & $\mathrm{C}-\mathrm{F}$ & 54483.82 & $1.811 \pm 0.018$ & \pm 0.37 & $\mathrm{n}$ & $\ldots$ & $4.55 \pm 0.20$ & & \\
\hline & & A-D & 54483.82 & $6.956 \pm 0.016$ & $105.37 \pm 0.11$ & $\mathrm{n}$ & $\ldots$ & $7.11 \pm 0.05$ & 4.91 & ${ }^{a} 4.80$ \\
\hline & & A-E & 54483.82 & $19.308 \pm 0.017$ & $63.53 \pm 0.05$ & $\mathrm{n}$ & $\ldots$ & $7.10 \pm 0.08$ & 5.23 & 4.96 \\
\hline & & $A-G$ & 54483.82 & $9.284 \pm 0.017$ & $20.48 \pm 0.09$ & $\mathrm{n}$ & $\ldots$ & $8.57 \pm 0.09$ & ${ }^{a} 5.26$ & ${ }^{a} 5.21$ \\
\hline & & A-H & 54483.82 & $9.984 \pm 0.017$ & $343.09 \pm 0.09$ & $\mathrm{n}$ & $\ldots$ & $8.73 \pm 0.08$ & ${ }^{a} 6.24$ & ${ }^{a} 6.28$ \\
\hline \multirow[t]{5}{*}{ HD 18326} & $02594+6034$ & A-B & 54483.89 & $2.451 \pm 0.144$ & $355.97 \pm 3.14$ & $\mathrm{y}$ & $\ldots$ & $8.15 \pm 1.34$ & & \\
\hline & & $\mathrm{A}-\mathrm{C}$ & 54483.89 & $8.039 \pm 0.016$ & $353.98 \pm 0.10$ & $\mathrm{n}$ & $\ldots$ & $5.00 \pm 0.04$ & 4.54 & 4.59 \\
\hline & & A-D & 54483.89 & $12.443 \pm 0.016$ & $327.28 \pm 0.07$ & $\mathrm{n}$ & & $5.72 \pm 0.05$ & 5.08 & 5.19 \\
\hline & & A-E & 54483.89 & $8.371 \pm 0.016$ & $248.60 \pm 0.09$ & $\mathrm{n}$ & $\ldots$ & $6.03 \pm 0.09$ & 5.02 & 5.00 \\
\hline & & E-F & 54483.89 & $0.992 \pm 0.016$ & $24.54 \pm 0.35$ & $\mathrm{n}$ & $\ldots$ & $0.57 \pm 0.12$ & $\ldots$ & \\
\hline HD 24431 & $03556+5238$ & A-B & 54483.91 & $0.720 \pm 0.016$ & $177.00 \pm 0.37$ & $\mathrm{y}$ & $\ldots$ & $2.80 \pm 0.02$ & & \\
\hline NSV 1458 - SZ Cam & $04078+6220$ & A-B & 54760.15 & $6.835 \pm 0.016$ & $256.38 \pm 0.11$ & $\mathrm{y}$ & $\ldots$ & $5.59 \pm 0.02$ & 3.98 & ${ }^{a} 3.74$ \\
\hline & & $\mathrm{A}-\mathrm{C}$ & 54760.15 & $11.173 \pm 0.017$ & $0.02 \pm 0.08$ & $\mathrm{y}$ & $\ldots$ & $4.56 \pm 0.02$ & 4.08 & 4.03 \\
\hline & & A-D & 54760.15 & $14.450 \pm 0.018$ & $131.92 \pm 0.07$ & $\mathrm{y}$ & & $6.29 \pm 0.02$ & 5.55 & 5.50 \\
\hline & & $\mathrm{Ea}-\mathrm{Eb}$ & 54483.91 & $0.103 \pm 0.016$ & $=1.86$ & $\mathrm{y}$ & & $0.02 \pm 0.08$ & & \\
\hline & & Ea-A & 54483.91 & $17.840 \pm 0.017$ & $124.79 \pm 0.05$ & $\mathrm{y}$ & -0.03 & $-1.03 \pm 0.54$ & -0.15 & -0.13 \\
\hline & & Ea-B & 54483.91 & $14.241 \pm 0.017$ & $145.84 \pm 0.06$ & $\mathrm{y}$ & $\ldots$ & $4.86 \pm 0.31$ & 3.83 & ${ }^{a} 3.60$ \\
\hline & & $\mathrm{Ea}-\mathrm{C}$ & 54483.91 & $14.674 \pm 0.017$ & $86.21 \pm 0.06$ & $\mathrm{y}$ & $\ldots$ & $4.03 \pm 0.30$ & 3.93 & 3.90 \\
\hline HD 34656 & $05207+3726$ & $\mathrm{Aa}-\mathrm{Ab}$ & 54483.92 & $0.373 \pm 0.017$ & $276.24 \pm 0.94$ & $\mathrm{y}$ & $\ldots$ & $3.13 \pm 0.07$ & $\ldots$ & \\
\hline & & Aa-B & 54483.92 & $1.948 \pm 0.018$ & $48.49 \pm 0.36$ & $\mathrm{y}$ & & $7.51 \pm 0.19$ & & \\
\hline & & Aa-E & 54483.92 & $8.683 \pm 0.016$ & $12.83 \pm 0.09$ & $\mathrm{y}$ & & $6.77 \pm 0.04$ & & \\
\hline HDE 242926 & $05227+3319$ & A-B & 54483.99 & $1.693 \pm 0.019$ & $171.86 \pm 0.33$ & $\mathrm{n}$ & & $4.89 \pm 0.23$ & $\ldots$ & \\
\hline HDE 242935 & $05228+3325$ & $A-B$ & 54483.99 & $1.081 \pm 0.016$ & $194.60 \pm 0.32$ & $\mathrm{y}$ & 0.77 & $0.80 \pm 0.01$ & & \\
\hline & & $\mathrm{A}-\mathrm{C}$ & 54483.99 & $9.736 \pm 0.016$ & $114.75 \pm 0.08$ & $\mathrm{y}$ & $\ldots$ & $3.30 \pm 0.01$ & 3.38 & 3.39 \\
\hline & & A-D & 54483.99 & $10.512 \pm 0.016$ & $332.65 \pm 0.08$ & $\mathrm{y}$ & & $2.53 \pm 0.01$ & 2.55 & 2.51 \\
\hline HD 35619 & $05276+3445$ & A-B & 54484.00 & $2.772 \pm 0.016$ & $307.29 \pm 0.20$ & $\mathrm{y}$ & & $2.88 \pm 0.01$ & $\ldots$ & \\
\hline LY Aur & $05297+3523$ & $A-B$ & 54483.92 & $0.598 \pm 0.016$ & $253.69 \pm 0.39$ & $\mathrm{y}$ & & $1.87 \pm 0.02$ & & \\
\hline$\delta$ Ori & $05320-0018$ & $\mathrm{Aa}-\mathrm{Ab}$ & 54482.84 & $0.325 \pm 0.016$ & $132.66 \pm 0.47$ & $\mathrm{y}$ & 1.35 & $1.48 \pm 0.02$ & & \\
\hline HD 36483 & $05337+3628$ & A-B & 54484.01 & $9.900 \pm 0.016$ & $265.53 \pm 0.08$ & $\mathrm{y}$ & $\ldots$ & $4.81 \pm 0.01$ & 4.24 & 4.18 \\
\hline$\lambda$ Ori & $05351+0956$ & A-B & 54482.90 & $4.342 \pm 0.016$ & $43.80 \pm 0.16$ & $\mathrm{y}$ & 1.94 & $1.91 \pm 0.02$ & $\ldots$ & \\
\hline$\theta^{1}$ Ori & $05353-0523$ & C-A & 54487.04 & $12.858 \pm 0.019$ & $311.84 \pm 0.08$ & $\mathrm{y}$ & 1.49 & $1.64 \pm 0.05$ & 1.17 & 1.27 \\
\hline & & C-Ba & 54487.04 & $16.855 \pm 0.021$ & $342.80 \pm 0.07$ & $\mathrm{y}$ & ${ }^{a} 2.43$ & $3.02 \pm 0.04$ & 1.67 & 1.60 \\
\hline
\end{tabular}


Table 3. Continued.

\begin{tabular}{|c|c|c|c|c|c|c|c|c|c|c|}
\hline System name & WDS & Pair & MJD & $\begin{array}{c}\text { Separation } \\
\left({ }^{\prime \prime}\right)\end{array}$ & $\begin{array}{c}\begin{array}{c}\text { Orientation } \\
\text { (degrees) }\end{array} \\
\end{array}$ & $\begin{array}{c}\text { pair } \\
\text { in WDS? }\end{array}$ & $\begin{array}{c}\Delta V_{\text {Ty }} \\
\text { (mag.) }\end{array}$ & $\begin{array}{c}\Delta z \\
\text { (mag.) }\end{array}$ & $\begin{array}{l}\Delta H_{2 \mathrm{M}} \\
\text { (mag.) }\end{array}$ & $\begin{array}{l}\Delta K_{2 \mathrm{M}} \\
\text { (mag.) }\end{array}$ \\
\hline & & $\mathrm{Ba}-\mathrm{Bb}$ & 54487.04 & $0.996 \pm 0.016$ & $252.63 \pm 0.36$ & $\mathrm{y}$ & & $3.02 \pm 0.02$ & & \\
\hline & & C-D & 54487.04 & $13.401 \pm 0.019$ & $61.86 \pm 0.08$ & $\mathrm{y}$ & 1.32 & $1.49 \pm 0.07$ & 1.26 & 1.35 \\
\hline & & C-E & 54487.04 & $16.630 \pm 0.021$ & $321.42 \pm 0.07$ & $\mathrm{y}$ & $\ldots$ & $3.63 \pm 0.05$ & 1.52 & 1.58 \\
\hline & & $\mathrm{C}-\mathrm{H}$ & 54487.04 & $9.426 \pm 0.018$ & $272.47 \pm 0.10$ & $\mathrm{y}$ & $\ldots$ & $6.81 \pm 0.12$ & 3.93 & 3.49 \\
\hline & & H-I & 54487.04 & $1.547 \pm 0.017$ & $270.16 \pm 0.35$ & $\mathrm{y}$ & $\ldots$ & $0.34 \pm 0.21$ & $\ldots$ & $\cdots$ \\
\hline & & C-F & 54487.04 & $4.555 \pm 0.016$ & $120.70 \pm 0.16$ & $\mathrm{y}$ & $\ldots$ & $4.72 \pm 0.17$ & & \\
\hline & & C-G & 54487.04 & $7.731 \pm 0.018$ & $33.28 \pm 0.12$ & $\mathrm{y}$ & $\ldots$ & $7.80 \pm 0.05$ & ${ }^{a} 4.26$ & 4.05 \\
\hline & & $C-Z$ & 54487.04 & $6.879 \pm 0.018$ & $337.04 \pm 0.13$ & $\mathrm{n}$ & $\ldots$ & $7.66 \pm 0.04$ & $\ldots$ & $\cdots$ \\
\hline$\theta^{2}$ Ori & 05354-0525 & $A a-A b$ & 54482.92 & $0.396 \pm 0.016$ & $295.12 \pm 0.56$ & $\mathrm{y}$ & $\ldots$ & $2.62 \pm 0.04$ & & \\
\hline$\iota$ Ori & $05354-0555$ & A-B & 54482.94 & $11.319 \pm 0.018$ & $141.14 \pm 0.08$ & $\mathrm{y}$ & $\ldots$ & $4.51 \pm 0.01$ & ${ }^{a} 3.96$ & ${ }^{a} 3.95$ \\
\hline HD 36879 & $05357+2124$ & A-B & 54484.02 & $9.387 \pm 0.016$ & $267.46 \pm 0.09$ & $\mathrm{n}$ & $\ldots$ & $3.31 \pm 0.01$ & 1.47 & 1.20 \\
\hline \multirow[t]{4}{*}{$\sigma$ Ori } & 05387-0236 & A-B & 54482.83 & $0.260 \pm 0.016$ & $94.47 \pm 0.59$ & $\mathrm{y}$ & $\ldots$ & $1.57 \pm 0.30$ & & \\
\hline & & A-C & 54482.83 & $11.443 \pm 0.018$ & $237.89 \pm 0.08$ & $\mathrm{y}$ & $\ldots$ & $5.23 \pm 0.29$ & ${ }^{a} 4.47$ & 4.64 \\
\hline & & A-D & 54482.83 & $13.031 \pm 0.019$ & $83.99 \pm 0.08$ & $\mathrm{y}$ & 2.79 & $3.05 \pm 0.28$ & ${ }^{a} 2.58$ & 2.77 \\
\hline & & $A-G$ & 54482.83 & $3.224 \pm 0.045$ & $20.34 \pm 0.65$ & $\mathrm{y}$ & $\ldots$ & $7.21 \pm 0.34$ & $\ldots$ & 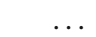 \\
\hline \multirow[t]{2}{*}{ HD 37366} & $05394+3053$ & A-B & 54483.07 & $0.593 \pm 0.016$ & $295.47 \pm 0.50$ & $\mathrm{y}$ & $\ldots$ & $3.73 \pm 0.15$ & & \\
\hline & & A-C & 54483.07 & $11.856 \pm 0.018$ & $44.29 \pm 0.08$ & $\mathrm{n}$ & & $4.29 \pm 0.02$ & 3.79 & 3.67 \\
\hline$\zeta$ Ori & $05407-0157$ & A-B & 54482.85 & $2.424 \pm 0.016$ & $165.70 \pm 0.22$ & $\mathrm{y}$ & 1.81 & $2.26 \pm 0.02$ & & \\
\hline HD 41161 & $06059+4815$ & A-B & 54484.08 & $10.277 \pm 0.016$ & $269.14 \pm 0.08$ & $\mathrm{y}$ & $\ldots$ & $5.41 \pm 0.02$ & 4.32 & 4.25 \\
\hline HDE 254755 & $06185+2241$ & A-B & 54761.14 & $0.188 \pm 0.016$ & $53.38 \pm 0.62$ & $\mathrm{y}$ & $\ldots$ & $2.09 \pm 0.05$ & & \\
\hline HD 44811 & $06246+1942$ & A-B & 54484.04 & $6.211 \pm 0.016$ & $139.95 \pm 0.12$ & $\mathrm{y}$ & ${ }^{a} 4.03$ & $3.71 \pm 0.01$ & 3.38 & 3.23 \\
\hline HD 46056 & $06313+0450$ & A-B & 54487.06 & $10.419 \pm 0.018$ & $336.50 \pm 0.09$ & $\mathrm{y}$ & $\ldots$ & $2.78 \pm 0.10$ & 2.48 & 2.46 \\
\hline \multirow[t]{4}{*}{ HD 46150} & $06319+0457$ & A-B & 54487.07 & $3.516 \pm 0.017$ & $286.53 \pm 0.20$ & $\mathrm{y}$ & $\ldots$ & $4.33 \pm 0.04$ & $\ldots$ & \\
\hline & & $\mathrm{A}-\mathrm{C}$ & 54487.07 & $6.853 \pm 0.017$ & $319.67 \pm 0.12$ & $\mathrm{y}$ & $\ldots$ & $5.98 \pm 0.10$ & & \\
\hline & & A-D & 54487.07 & $12.519 \pm 0.019$ & $289.42 \pm 0.08$ & $\mathrm{y}$ & $\ldots$ & $5.36 \pm 0.02$ & 4.53 & 4.43 \\
\hline & & A-L & 54487.07 & $10.404 \pm 0.018$ & $46.79 \pm 0.09$ & $\mathrm{n}$ & $\ldots$ & $6.29 \pm 0.11$ & 4.78 & 4.63 \\
\hline HD 46202 & $06321+0458$ & D-E & 54484.05 & $3.707 \pm 0.016$ & $262.90 \pm 0.18$ & $\mathrm{n}$ & $\ldots$ & $3.11 \pm 0.02$ & ${ }^{a} 0.69$ & 0.32 \\
\hline HD 47129 & $06374+0608$ & A-B & 54487.09 & $1.156 \pm 0.029$ & $252.48 \pm 1.11$ & $\mathrm{y}$ & $\ldots$ & $6.28 \pm 0.26$ & $\ldots$ & $\ldots$ \\
\hline HD 47032 & $06388+0442$ & A-B & 54761.15 & $3.490 \pm 0.016$ & $258.84 \pm 0.19$ & $\mathrm{n}$ & $\ldots$ & $5.80 \pm 0.06$ & $\ldots$ & $\ldots$ \\
\hline \multirow[t]{2}{*}{15 Mon } & $06410+0954$ & $A a-A b$ & 54482.93 & $0.128 \pm 0.016$ & $260.94 \pm 1.47$ & $\mathrm{y}$ & $\ldots$ & $1.43 \pm 0.13$ & $\ldots$ & $\ldots$ \\
\hline & & Aa-B & 54482.93 & $2.976 \pm 0.017$ & $213.50 \pm 0.21$ & $\mathrm{y}$ & 3.15 & $3.23 \pm 0.16$ & $\ldots$ & \\
\hline HD 48279 & $06427+0143$ & A-B & 54760.16 & $6.860 \pm 0.016$ & $193.85 \pm 0.11$ & $\mathrm{y}$ & 2.72 & $2.36 \pm 0.06$ & 1.62 & 1.56 \\
\hline \multirow[t]{3}{*}{ HD 52533} & 07015-0307 & A-B & 54761.16 & $2.676 \pm 0.019$ & $186.90 \pm 0.28$ & $\mathrm{y}$ & $\ldots$ & $4.89 \pm 0.07$ & & \\
\hline & & A-E & 54761.16 & $11.504 \pm 0.017$ & $305.64 \pm 0.08$ & $\mathrm{n}$ & $\ldots$ & $5.92 \pm 0.03$ & 4.99 & 4.83 \\
\hline & & A-F & 54761.16 & $11.281 \pm 0.017$ & $194.74 \pm 0.08$ & $\mathrm{n}$ & $\ldots$ & $5.78 \pm 0.03$ & 5.58 & 5.55 \\
\hline HD 167659 & 18170-1858 & A-B & 54963.15 & $17.531 \pm 0.034$ & $226.71 \pm 0.39$ & $\mathrm{n}$ & $\ldots$ & $6.10 \pm 0.02$ & 4.81 & 4.76 \\
\hline HD 167771 & $18175-1828$ & A-B & 54632.08 & $8.548 \pm 0.022$ & $168.78 \pm 0.40$ & $\mathrm{y}$ & $\ldots$ & $5.93 \pm 0.06$ & $\ldots$ & $\cdots$ \\
\hline HD 190429 & $20035+3601$ & A-B & 54631.95 & $1.959 \pm 0.016$ & $173.80 \pm 0.46$ & $\mathrm{y}$ & 0.64 & $0.61 \pm 0.01$ & $\ldots$ & $\ldots$ \\
\hline \multirow[t]{3}{*}{ WR 133} & $20060+3547$ & A-B & 54631.96 & $6.855 \pm 0.020$ & $63.36 \pm 0.40$ & $\mathrm{y}$ & $\ldots$ & $5.27 \pm 0.02$ & & \\
\hline & & A-C & 54631.96 & $12.394 \pm 0.027$ & $28.96 \pm 0.39$ & $\mathrm{y}$ & $\cdots$ & $3.46 \pm 0.01$ & 2.40 & 2.36 \\
\hline & & A-D & 54631.96 & $11.286 \pm 0.025$ & $300.13 \pm 0.39$ & $\mathrm{y}$ & 2.71 & $2.98 \pm 0.01$ & 3.00 & 3.09 \\
\hline \multirow{2}{*}{ HD 193322} & $20181+4044$ & $A a-A b$ & 54632.07 & $0.055 \pm 0.016$ & $119.81 \pm 2.47$ & $\mathrm{y}$ & 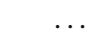 & $-0.04 \pm 0.19$ & $\ldots$ & $\ldots$ \\
\hline & & Aa-B & 54632.07 & $2.719 \pm 0.017$ & $244.97 \pm 0.44$ & $\mathrm{y}$ & 2.28 & $1.52 \pm 0.10$ & $\ldots$ & $\ldots$ \\
\hline WR 140 & $20205+4351$ & A-B & 54632.09 & $4.815 \pm 0.018$ & $210.94 \pm 0.41$ & $\mathrm{y}$ & $\ldots$ & $6.56 \pm 0.04$ & $\cdots$ & \\
\hline \multirow[t]{2}{*}{ Cyg OB2-4 } & $20322+4127$ & A-B & 54417.86 & $14.607 \pm 0.030$ & $327.09 \pm 0.39$ & $\mathrm{n}$ & $\ldots$ & $1.96 \pm 0.01$ & 2.00 & 1.97 \\
\hline & & A-C & 54417.86 & $18.265 \pm 0.035$ & $348.72 \pm 0.39$ & $\mathrm{n}$ & & $3.17 \pm 0.01$ & 3.12 & 3.08 \\
\hline \multirow[t]{3}{*}{ Cyg OB2-5 } & $20324+4118$ & A-B & 54417.78 & $0.934 \pm 0.016$ & $54.42 \pm 0.53$ & $\mathrm{y}$ & ${ }^{a} 2.55$ & $3.02 \pm 0.03$ & & \\
\hline & & A-C & 54417.78 & $20.472 \pm 0.039$ & $323.18 \pm 0.39$ & $\mathrm{n}$ & $\ldots$ & $6.02 \pm 0.02$ & 6.20 & 6.33 \\
\hline & & A-D & 54417.78 & $5.532 \pm 0.020$ & $225.25 \pm 0.41$ & $\mathrm{n}$ & $\cdots$ & $7.38 \pm 0.15$ & $\ldots$ & \\
\hline \multirow[t]{9}{*}{ Cyg OB2-22 } & $20331+4113$ & $\mathrm{~A}-\mathrm{Ba}$ & 54416.78 & $1.536 \pm 0.016$ & $145.88 \pm 0.48$ & $\mathrm{y}$ & $\cdots$ & $0.55 \pm 0.04$ & $\ldots$ & \\
\hline & & $\mathrm{Ba}-\mathrm{Bb}$ & 54416.78 & $0.257 \pm 0.020$ & $179.89 \pm 2.72$ & $\mathrm{n}$ & $\ldots$ & $3.13 \pm 0.50$ & $\ldots$ & $\ldots$ \\
\hline & & $\mathrm{A}-\mathrm{C}$ & 54416.78 & $20.398 \pm 0.039$ & $151.85 \pm 0.39$ & $\mathrm{n}$ & $\ldots$ & $1.32 \pm 0.03$ & 1.60 & 1.54 \\
\hline & & C-D & 54416.78 & $11.179 \pm 0.025$ & $30.92 \pm 0.39$ & $\mathrm{n}$ & $\ldots$ & $0.51 \pm 0.03$ & 0.77 & 0.86 \\
\hline & & C-E & 54416.78 & $2.822 \pm 0.017$ & $218.05 \pm 0.44$ & $\mathrm{n}$ & $\ldots$ & $1.69 \pm 0.02$ & $\ldots$ & $\ldots$ \\
\hline & & E-F & 54416.78 & $1.864 \pm 0.017$ & $189.13 \pm 0.48$ & $\mathrm{n}$ & $\ldots$ & $1.77 \pm 0.10$ & $\ldots$ & $\ldots$ \\
\hline & & C-G & 54416.78 & $3.862 \pm 0.017$ & $31.90 \pm 0.42$ & $\mathrm{n}$ & $\ldots$ & $3.93 \pm 0.05$ & & \\
\hline & & $\mathrm{C}-\mathrm{H}$ & 54416.78 & $7.892 \pm 0.021$ & $101.71 \pm 0.40$ & $\mathrm{n}$ & $\ldots$ & $4.27 \pm 0.04$ & 4.31 & 4.34 \\
\hline & & C-I & 54416.78 & $10.416 \pm 0.025$ & $58.46 \pm 0.39$ & $\mathrm{n}$ & $\ldots$ & $4.56 \pm 0.06$ & $\cdots$ & \\
\hline Cyg OB2-9 & $20332+4115$ & A-B & 54417.82 & $21.005 \pm 0.040$ & $106.35 \pm 0.39$ & $\mathrm{n}$ & $\ldots$ & $6.41 \pm 0.03$ & 5.31 & 4.86 \\
\hline Cyg OB2-7 & $20332+4120$ & A-B & 54417.87 & $18.623 \pm 0.036$ & $104.23 \pm 0.39$ & $\mathrm{n}$ & $\ldots$ & $2.05 \pm 0.01$ & 2.12 & 2.11 \\
\hline & & A-C & 54417.87 & $15.676 \pm 0.032$ & $106.03 \pm 0.39$ & $\mathrm{n}$ & $\ldots$ & $5.87 \pm 0.03$ & & \\
\hline & & A-D & 54417.87 & $20.400 \pm 0.039$ & $79.06 \pm 0.39$ & $\mathrm{n}$ & $\cdots$ & $5.93 \pm 0.06$ & 5.18 & ${ }^{a} 5.11$ \\
\hline Cyg OB2-8 & $20333+4119$ & A-B & 54417.83 & $9.441 \pm 0.023$ & $202.11 \pm 0.39$ & $\mathrm{y}$ & 1.57 & $1.29 \pm 0.01$ & 1.04 & 1.07 \\
\hline & & A-D & 54417.83 & $18.182 \pm 0.035$ & $50.77 \pm 0.39$ & $\mathrm{y}$ & & $2.96 \pm 0.01$ & 2.70 & 2.74 \\
\hline & & C-E & 54417.84 & $10.353 \pm 0.024$ & $184.45 \pm 0.39$ & $\mathrm{n}$ & & $5.27 \pm 0.02$ & ${ }^{a} 4.82$ & 4.91 \\
\hline LS III +46 11 & $20352+4651$ & A-B & 54417.91 & $22.563 \pm 0.042$ & $139.77 \pm 0.39$ & $\mathrm{n}$ & $\ldots$ & $5.28 \pm 0.02$ & 4.86 & ${ }^{b}-$ \\
\hline
\end{tabular}


Table 3. Continued.

\begin{tabular}{|c|c|c|c|c|c|c|c|c|c|c|}
\hline System name & WDS & Pair & MJD & $\begin{array}{c}\text { Separation } \\
\left({ }^{\prime \prime}\right)\end{array}$ & $\begin{array}{c}\text { Orientation } \\
\text { (degrees) }\end{array}$ & $\begin{array}{c}\text { pair } \\
\text { in WDS? }\end{array}$ & $\begin{array}{c}\Delta V_{\mathrm{Ty}} \\
\text { (mag.) }\end{array}$ & $\begin{array}{c}\Delta z \\
\text { (mag.) }\end{array}$ & $\begin{array}{l}\Delta H_{2 \mathrm{M}} \\
\text { (mag.) }\end{array}$ & $\begin{array}{l}\Delta K_{2 \mathrm{M}} \\
\text { (mag.) }\end{array}$ \\
\hline & & $\mathrm{A}-\mathrm{C}$ & 54417.91 & $14.259 \pm 0.030$ & $114.21 \pm 0.39$ & $\mathrm{n}$ & & $6.13 \pm 0.04$ & 5.65 & $b_{-}$ \\
\hline HD 206267 & $21390+5729$ & $\mathrm{~A}-\mathrm{C}$ & 54417.93 & $11.824 \pm 0.026$ & $119.31 \pm 0.39$ & $\mathrm{y}$ & 1.75 & $2.52 \pm 0.01$ & 2.38 & 2.38 \\
\hline HD 210809 & $22116+5226$ & A-B & 54761.06 & $5.858 \pm 0.017$ & $333.41 \pm 0.13$ & $\mathrm{n}$ & $\ldots$ & $6.06 \pm 0.04$ & 5.20 & 5.21 \\
\hline WR 153ab & $22188+5608$ & A-B & 54761.07 & $10.058 \pm 0.017$ & $35.98 \pm 0.09$ & $\mathrm{n}$ & 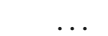 & $7.11 \pm 0.05$ & ${ }^{a} 6.71$ & 6.77 \\
\hline DH Cep & $22469+5805$ & A-B & 54761.07 & $7.307 \pm 0.017$ & $246.69 \pm 0.11$ & $\mathrm{n}$ & & $7.15 \pm 0.06$ & $\ldots$ & \\
\hline \multirow[t]{3}{*}{ HD 217086} & $22568+6244$ & A-B & 54761.05 & $2.865 \pm 0.016$ & $355.05 \pm 0.20$ & $\mathrm{y}$ & 3.58 & $3.58 \pm 0.02$ & $\ldots$ & $\ldots$ \\
\hline & & A-C & 54761.05 & $3.261 \pm 0.017$ & $166.22 \pm 0.23$ & $\mathrm{y}$ & $\ldots$ & $6.40 \pm 0.09$ & & \\
\hline & & A-D & 54761.05 & $11.395 \pm 0.017$ & $52.71 \pm 0.08$ & $\mathrm{n}$ & & $7.78 \pm 0.06$ & 6.48 & ${ }^{a} 6.04$ \\
\hline \multirow[t]{3}{*}{ HD 218195} & $23052+5815$ & A-B & 54761.08 & $0.919 \pm 0.016$ & $80.49 \pm 0.35$ & $\mathrm{y}$ & 2.64 & $2.56 \pm 0.02$ & $\cdots$ & \\
\hline & & $\mathrm{A}-\mathrm{C}$ & 54761.08 & $3.545 \pm 0.017$ & $304.60 \pm 0.21$ & $\mathrm{n}$ & $\ldots$ & $5.65 \pm 0.06$ & & \\
\hline & & A-D & 54761.08 & $11.524 \pm 0.017$ & $154.05 \pm 0.08$ & $\mathrm{n}$ & $\cdots$ & $6.46 \pm 0.02$ & 5.85 & 5.80 \\
\hline
\end{tabular}

Notes. ${ }^{(a)}$ Large (>0.2 mag) photometric uncertainty; ${ }^{(b)}$ Low photometric quality (X flag). 